\title{
Self-amplified coherent spontaneous emission in a free electron laser with "quiet" bunches
}

\author{
V. A. Goryashko* and V. Ziemann \\ Uppsala University, Sweden \\ (Received 15 May 2012; published 12 March 2013)
}

\begin{abstract}
For a planar free electron laser (FEL) configuration we study self-amplified coherent spontaneous emission driven by a gradient of the bunch current in the presence of different levels of noise in bunches. The longitudinal granularity of the electron bunch density originating from shot noise is maintained throughout the analysis. For the FEL model with the SwissFEL injector bunch parameters, we calculate the probability density distribution of the maximum power of the radiation pulses for different levels of shot noise. It turns out that the temporal coherence quickly increases as the noise level reduces. We also show that the FEL based on coherent spontaneous emission produces almost Fourier transform limited pulses. The analysis indicates that the time-bandwidth product is mainly determined by the bunch length and the interaction distance in an undulator. Calculations of the FEL characteristics for different rise times of the front of the current pulse are performed, and it is found that a reduced level of the power fluctuations is preserved for the bunch current pulse with a front duration up to several FEL wavelengths. We also propose a novel scheme that permits the formation of electron bunches with a reduced level of noise and a high gradient of the current at the bunch tail to enhance coherent spontaneous emission. The presented scheme uses effects of noise reduction and controlled microbunching instability and consists of a laser heater, a bunch compressor, and a shot noise suppression section. We show that shot noise reduction by 2 orders of magnitude in electron bunches produced by the SwissFEL injector can be achieved in a compact noise suppression section. The noise factor and microbunching gain of the overall proposed scheme with and without laser heater are estimated.
\end{abstract}

DOI: 10.1103/PhysRevSTAB.16.030702

PACS numbers: 41.60.Cr, 29.27.-a, 41.85.-p, 41.60.Dk

\section{INTRODUCTION}

Operating free electron lasers in the vacuum ultraviolet (VUV) and x-ray regions are usually based on the self-amplified spontaneous emission (SASE) process starting from spontaneous emission of undulator radiation initiated by shot noise [1]. The latter is a stochastic process and the SASE free electron laser (FEL) produces a series of random superradiant spikes with a large variation of intensities [2]. A method of direct FEL seeding by a coherent quantum laser operating in higher harmonic generation regime as well as methods of highgain and echo-enabled harmonic generation in FELs has been proposed to overcome this deficiency [3-5]. Recent experiments on self-seeded FEL using $\mathrm{x}$ rays from the first half of the undulator to seed the second half through a diamond-based monochromator [6] and FEL operating in a SASE mode with a chirped electron beam and a tapered undulator [7] showed a substantial reduction of the relative FEL bandwidth. However, the stability of the final seeded FEL power in [6] is still poor $(50 \% \mathrm{rms}$

\footnotetext{
*vitaliy.goryashko@physics.uu.se vitgor06@gmail.com

Published by the American Physical Society under the terms of the Creative Commons Attribution 3.0 License. Further distribution of this work must maintain attribution to the author(s) and the published article's title, journal citation, and DOI.
}

fluctuations), partly due to shot-to-shot electron energy variations. Apart from external seeding, intrinsic seeding originating from coherent spontaneous emission (CSE) driven by the current gradient of electron bunches can be employed to obtain highly coherent radiation [8-11]. However, shot noise competes with the coherent seeding and reduces coherence of the radiation pulses and pulseto-pulse reproducibility.

To aid the FEL process, the authors of [12-14] have proposed schemes to decrease the noise level below the shot noise level resulting in the so-called "quiet" bunches. Specifically, a space-charge dominated interaction region followed by a dispersion section suppresses shot noise over a wide range of frequencies (the experiment [15] demonstrated that after a $1 / 4$ plasma oscillation period the initial periodic terahertz modulation is completely washed out and one obtains a uniform temporal profile of the bunch density as predicted in [12]). Recently, the shot noise suppression effect in the optical wave range was demonstrated experimentally by measuring a decrease in the optical transition radiation (OTR) emitted from a downstream foil $[16,17]$. OTR intensity was suppressed by $30 \%-35 \%$ of the shot noise level. It is expected that the noise factor of the bunch distribution is suppressed more strongly than the observed OTR. Impact of the shot noise suppression on the FEL performance is currently under study and the analysis of the linear regime [13,18-21] shows that high coherence and spectral brightness of 
seeded FELs operating at optical frequencies and the VUV region is possible.

In view of the shot noise reduction schemes, the intrinsic seeding driven by the current gradient of quiet electron bunches becomes an attractive way of obtaining pulse-topulse stable stimulated CSE radiation without external seeding lasers. The temporal structure of the radiation pulses is predetermined by preparing electron bunches with a special current distribution such that amplified radiation pulses will exhibit the reproducibility of the electron bunch current [22]. For example, in the experiment [23] very stable pulse-to-pulse radiation signals were observed from quasirectangular bunches with steep rise and fall of the particle distributions.

Electromagnetic pulses with well-determined temporal structure are of interest for coherent scattering, ultrafast spectroscopy, and nonlinear X-ray science [24]. One should mention once again that the longitudinally coherent radiation in FELs can also be obtained by employing seeding techniques. However, straightforward application of seeding techniques allows one to realize radiation only for a discrete set of frequencies dependent on the frequencies of lasers used for the beam modulation or determined by the properties of a monochromator in the self-seeding scheme, respectively. Therefore, a great advantage of generation of wavelength continuously tunable radiation is lost in FELs based on seeding schemes. This drawback of the seeding schemes can be avoided by using optical parametric amplification [25] that allows one to change the wavelength of the seed laser at some spectral range such that coherent FEL radiation in a continuous (spectral) range can be obtained [26]. The seeding schemes typically require the laser energy to be up to several millijoules [27] that is achievable by commercially available optical parametric amplifier (OPAs). At the same time, the capability to use OPAs for the FEL seeding with a high repetition rate is questionable. For example, to reach the output energy from an OPA sufficient to drive a FEL, the optical pump laser has to produce at least $100 \mathrm{~mJ}$. In view of a tendency towards FELs based on superconducting technology [28] like the European XFEL and next generation light source, the energy of a pump laser to drive an OPA has to be a few $\mathrm{kJ}$ per second. This implies a pump laser system that is well beyond the existing state-of-the-art lasers to the author's knowledge. One can operate an OPA at a lower repetition rate but then the advantage of the superconducting technology will not be used to a full extent.

One should also mention that a high-gain harmonic generation FEL can be made tunable by applying the techniques proposed in [29-31]. They propose the tunable seeded FEL wherein the seeds wavelength is fixed and the variations in the wavelength of radiation are achieved by compressing the bunch after seeding. The two-chicane wavelength compression of seeded FELs is proposed as well [32]. However, the seeded FEL is sensitive to the seed laser phase/wave front errors [33] so that rigid requirement will be imposed on seed laser characteristics and development of seeded FELs is a challenge [27]. Thus, a FEL based self-amplified CSE and making use of quiet bunches is an attractive alternative to seeded FELs in the VUV. The goal of the present paper is to investigate the self-amplified CSE in a FEL in the presence of different levels of noise and determine the level of reduction of shot noise required to obtain radiation pulses with a well-determined temporal structure.

In order to initiate strong CSE, the current gradients of the electron bunch have to be sufficiently large; this implies that the distribution at the tail of a bunch has to have a steep fall. In addition, in order to achieve complete dominance of a coherent emission over an incoherent one, the shot noise level in the electron bunch has to be reduced. To create bunches with the density distribution meeting the mentioned requirements, we propose a novel bunch formation scheme that uses the effects of noise suppression and controlled microbunching instability [34,35]. In our scheme bunches from the injector pass successively through a laser heater, a bunch compressor, and a noise suppression section. The latter is used to obtain quiet bunches whereas the former two are employed to achieve a high current gradient at the bunch tail using the controlled microbunching instability in a way similar to that used in the longitudinal space-charge amplifier (LSCA) [36]. The principal difference from an LSCA is that only the tail of the bunch is allowed to be sensitive to the microbunching instability whereas the main core of the bunch has to be stable against the instability. This can be realized by using a laser heater [37,38] with a partial overlap between electron and laser pulses such that the bunch tail remains unheated. Then, after passing through the magnetic compressor the bunch tail will have a sharp edge with a scale of the current gradient variation of the order of the variation of the laser used for heating. Note that magnetic bunch compression used in [23] resulted in steep variations of the bunch particle density and allowed coherent spontaneous emission to dominate over the incoherent emission. A detailed study of the formation of electron bunches with special density distribution is beyond the scope of the present study, and we will limit ourselves to order-of-magnitude analytical estimates of the bunch properties as the bunch passes through the proposed formation scheme.

The paper is organized as follows. In Sec. II we discuss the startup of coherent spontaneous emission and give a solution to the problem of excitation of the radiation field. The self-consistent nonaveraged FEL model is briefly formulated in Sec. III. In the following section we study the characteristics of a FEL driven by a gradient of the bunch current in the presence of different levels of noise in bunches. Formation of electron bunches with a reduced level of noise and a high current gradient at the bunch tail is 
considered in Sec. V. The obtained results are summarized in the Conclusion, and, finally, the numerical solution to the self-consistent FEL model and the feasibility of FELs based on CSE in the VUV region are discussed in Appendices A and B, respectively.

\section{FORMATION OF COHERENT SPONTANEOUS EMISSION}

We discuss the startup of CSE using a 1D approximation for the electron bunch but still consider the longitudinal discreteness of the electrons. Within this approximation electrons can be treated as charged disks with charge per unit area $e / S_{b}(e<0)$, where $e$ and $S_{b}$ are the electron charge and bunch transverse size, respectively. The dependence of the bunch current and excited radiation field on the transverse coordinates is ignored in this approach and evolution of the radiation field is governed by the $1 \mathrm{D}$ wave equation. Let us label the longitudinally discrete sources of radiation by the index $q=1, \ldots, Q_{b}$, where $Q_{b}$ is the total number of electrons in the bunch. Choosing the axial coordinate $z$ to be the independent variable, we denote the arrival time of the $q$ th electron at $z$ by $t_{q}(z)$ and the transverse and longitudinal velocities by $v_{\perp \mid q}(z)$ and $v_{z \mid q}$, respectively. Then, the transverse current density created by the wiggling electron bunch reads

$$
j_{\perp}(z, t)=\frac{e}{S_{b}} \sum_{q}^{Q_{b}} \frac{v_{\perp \mid q}(z)}{v_{z \mid q}(z)} \delta\left[t-t_{q}(z)\right],
$$

where $\delta(z)$ is the Dirac delta function, and the transverse velocity in a planar undulator with period $k_{u}$ and on-axis magnetic field magnitude $B_{u}$ may be written as

$$
v_{\perp \mid q}(z)=\left(\mathcal{K}_{c} / \gamma_{q}\right) \sin \left[k_{u} z\right] .
$$

Here $\mathcal{K}=|e| B_{u} / m_{e} c^{2} k_{u}$ is the undulator parameter, $\gamma_{q}$ is the energy of the $q$ th electron in units of its rest mass $m_{e}$, and $c$ is the velocity of light. In a planar undulator electrons move only in one plane, and we use scalar notation for the transverse current density and the radiation field throughout the paper.

In a FEL the incoherent undulator radiation from relativistic electrons is about $\gamma^{4}$ times larger in the forward direction (with respect to the bunch propagation) than in the backward direction, where $\gamma$ is the average bunch energy in units of the rest mass. In the case of selfamplified emission we only consider the forward radiation because it is strongly dominant. Thus, the transverse component of the vector-potential excited by current $j_{\perp}$ and copropagating with bunches is

$$
A_{\perp}(z, t)=\frac{1}{c} \int_{-T_{b}}^{t} d t^{\prime} \int_{0}^{z} d z^{\prime} G\left(z-z^{\prime}, t-t^{\prime}\right) j_{\perp}\left(z^{\prime}, t^{\prime}\right),
$$

where $G\left(z-z^{\prime}, t-t^{\prime}\right)$ is the Green function of the 1D wave equation and given by [39]. It is given by [39]

$$
G\left(z-z^{\prime}, t-t^{\prime}\right)=2 \pi c U\left[c\left(t-t^{\prime}\right)-\left(z-z^{\prime}\right)\right] .
$$

Here, $T_{b}$ is the bunch duration (electrons enter the interaction region during the time interval from $-T_{b}$ to 0 ) and $U[t]$ is the unit-step function. Note that the radiation emitted only at time $t-\left(z-z^{\prime}\right) / c$ by electrons in position $z^{\prime}$ contributes to the radiation observed at position $z$ and time $t$ because of causality. Using Eqs. (1)-(4) after some algebra we get the electric field of the form

$$
\begin{aligned}
E_{\perp}(z, t) \equiv & -\frac{1}{c} \frac{\partial A_{\perp}}{\partial t} \\
= & -\frac{2 \pi e}{S_{b}} \sum_{q}^{Q_{b}} \int_{0}^{z} \frac{v_{\perp \mid q}\left(z^{\prime}\right)}{v_{z \mid q}\left(z^{\prime}\right)} U\left[t-t_{q}\left(z^{\prime}\right)\right] U\left[t_{q}\left(z^{\prime}\right)+T_{b}\right] \\
& \times \delta\left\{\left[c t-c t^{\prime}\left(z^{\prime}\right)\right]-\left(z-z^{\prime}\right)\right\} d z^{\prime} .
\end{aligned}
$$

If the electron trajectories are dictated only by the undulator field such that $t_{q}(z) \approx t_{q}^{e}+z / \bar{v}_{z \mid q}$, where $\bar{v}_{z \mid q}$ is the average longitudinal velocity of the $q$ th electron, then from Eq. (5) we recover the well-known result [10]

$$
\begin{aligned}
E_{\perp}(z, t)= & -\frac{4 \pi e}{S_{b}} \sum_{q}^{Q_{b}} \frac{\mathcal{K} \gamma_{q}}{1+\mathcal{K}^{2} / 2} \sin \left[\omega_{q}\left(t-t_{q}^{e}-z / c\right)\right] \\
& \times U\left[c\left(t-t_{q}^{e}\right)-z\right] U\left[z-\bar{v}_{z \mid q}\left(t-t_{q}^{e}\right)\right],
\end{aligned}
$$

where $\omega_{q}=2 \gamma_{q}^{2} k_{u} \bar{v}_{z \mid q} /\left(1+\mathcal{K}^{2} / 2\right)$. Equation (6) provides immediate insight into the formation of coherent spontaneous emission in the FEL and is used later for verification of the numerical simulation scheme. We see that the electric field is the superposition of limited in space and time plane waves with different frequencies $\omega_{q}$ that depend on the electron energy. The plane wave produced by the $q$ th electron and observed at longitudinal position $z$ is nonzero only during the observation period from $t_{q}^{e}+z / c$ to $t_{q}^{e}+z / \bar{v}_{q \mid z}$. This result becomes obvious if we analyze the arrival time of photons emitted by a test electron. When the test electron enters the interaction region it emits photons that propagates with the velocity of light and leaves the electron behind such that photons slip ahead with respect to the electron by one radiation wavelength $\lambda_{r}$ in each undulator period $\lambda_{u}$. These photons are the first photons observed from the test electron. At the same time, the test electron continues to radiate during its passage through the interaction region such that the last photons will be observed when this electron passes through the observation point. Because emitted photons propagate faster than the electron, the radiation field observed at moment $t$ in position $z$ is created by photons emitted by the same electron but in different longitudinal positions. The total radiation field is a superposition of elementary radiation pulses from individual electrons; see Fig. 1 in which we schematically show electrons contributing to the observed field. Note that these elementary radiation pulses reach the observation point with different phases depending on the positions where they were emitted. The 


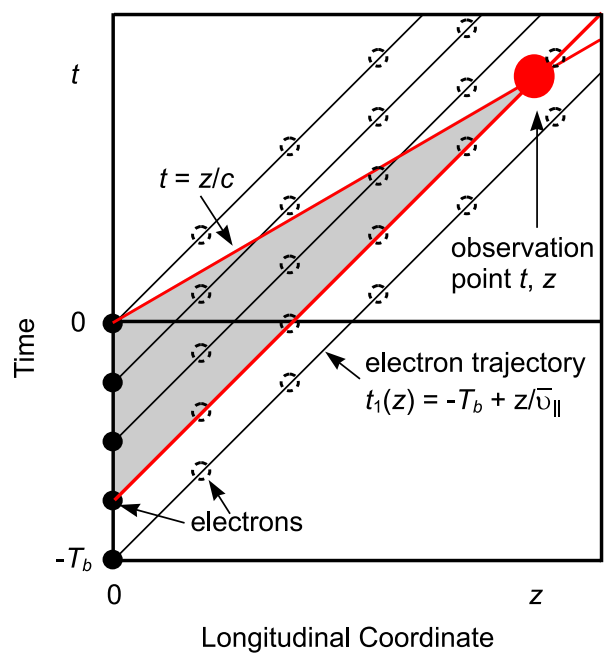

FIG. 1. The space-time diagram of the longitudinal dynamics of the bunch electrons in the undulator. The radiation field observed at moment $t$ and position $z$ is created by electrons passing through the shaded area.

number of electrons contributing to the observed field also depends on the observation position because the further away the observation position is, the more electrons can contribute due to the slippage.

Visual demonstration of the field pattern in the spacetime domain arising from the interference of elementary radiation pulses is complicated but the mathematical description following from Eq. (6) is straightforward. Specifically, for a monoenergetic electron bunch with a rectangular current profile, we can replace summation over electrons by integration over the electron entrance time in Eq. (6) such that the integration yields

$$
\begin{aligned}
E_{\perp}(z, t)= & -\frac{e}{S_{b}} \frac{2 \mathcal{K} \gamma}{1+\mathcal{K}^{2} / 2} \frac{\lambda_{r}}{c T_{b}}\left\{\cos \left[\frac{2 \pi}{\lambda_{r}}\left(c t-c t_{2}-z / c\right)\right]\right. \\
& \left.-\cos \left[\frac{2 \pi}{\lambda_{r}}\left(c t-c t_{1}-z / c\right)\right]\right\} U\left[t_{2}-t_{1}\right]
\end{aligned}
$$

where $t_{1}=\max \left[-T_{b}, t-z / \bar{v}_{\|}\right], t_{2}=\min \left[0, t-z / \bar{v}_{\|} \mid\right.$, and $\bar{v}_{\|}$is the average longitudinal velocity of the electrons. The radiation field created by moving and emitting electrons according to Eq. (7) is plotted in Fig. 2 at different longitudinal observation positions $z$ in the undulator. The results are shown in the frame moving with the velocity of bunch such that the bunch positions remain unchanged whereas the radiation field slips to the right.

Let us analyze the radiation pulses' shapes shown in Fig. 2 in detail for observation positions $z=\lambda_{u} / 2$ (top left) and $z=\lambda_{u}$ (middle left). In the first case the radiation field increases from the point $\mathrm{A}(s=0)$ to the point $\mathrm{B}\left(s=\lambda_{r} / 2\right)$ because more electrons contribute to the total field due to the slippage. The phase difference between the radiation coming from the point $\mathrm{A}$ and the radiation created in the point B is $\pi$ such that the fields created by

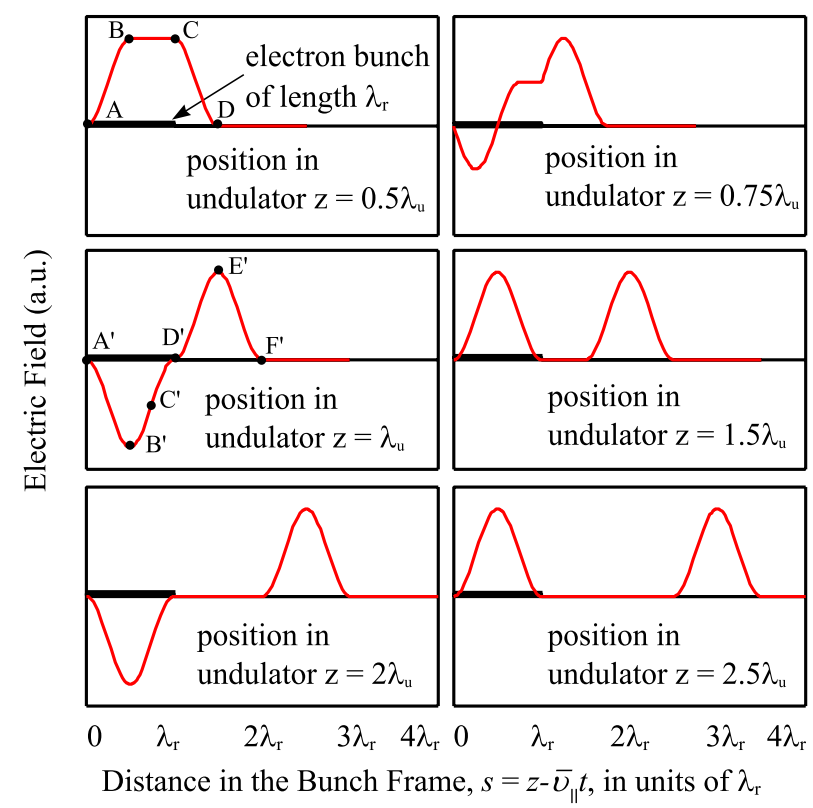

FIG. 2. The electric field created by the uniform electron bunch of length $\lambda_{r}$ as a function of distance, $s=z-\bar{v}_{\|} t$, in the frame moving with the bunch. Different cross sections of the undulator are shown. The arrival time of electrons at the observation position in the undulator is shown by the black box lying on the abscissa.

electrons located between the points A and B have the same sign and the field reaches its maximal value at the point $\mathrm{B}$. Between points $\mathrm{B}$ and $\mathrm{C}\left(s=\lambda_{r}\right)$ the number of contributing electrons as well as the maximal phase shift between them remain constant and the field does not change. After the point $\mathrm{C}$ the number of electrons contributing due to slippage decreases and the field decreases correspondingly.

At the undulator cross section $z=\lambda_{u}$ the field between the points $\mathrm{A}^{\prime}$ and $\mathrm{B}^{\prime}$ changes in the same way as in the case $z=\lambda_{u} / 2$ with the exception that radiation fields of all electrons contributing to the field between the points $A^{\prime}$ and $\mathrm{B}^{\prime}$ change the phase by $\pi$ such that the sign of the field magnitude is negative. At the point $\mathrm{D}^{\prime}$ the total field equals zero because fields radiated by electrons located in the regions $\mathrm{A}^{\prime} \mathrm{B}^{\prime}$ and $\mathrm{B}^{\prime} \mathrm{D}^{\prime}$ of the bunch are shifted by $\pi$ and the number of contributing electrons in these regions are equal. Therefore, these fields from individual electrons cancel. At the intermediate point $\mathrm{C}^{\prime}$ such cancellation does not happen.

\section{NONAVERAGED FEL MODEL}

Let us first give a brief formulation of the 1D selfconsistent nonaveraged model of the planar FEL that allows us to treat CSE correctly by preserving the longitudinal discreteness of the electron bunch in the equation for the radiation field. We will take 3D effects into account in a phenomenological way by redefining the FEL parameter in a manner similar to how it is done for the low-gain 
FEL [40]. Moreover, we introduce the traditional set of dimensionless variables [41]

$$
\zeta=\frac{z}{\ell_{g}}, \quad \tau=\frac{z-\bar{v}_{\|} t}{\ell_{g}\left(1-\bar{\beta}_{\|}\right)}, \quad F=\frac{1}{4} \frac{|e| E_{\perp}}{m_{e} c^{2}} \frac{\ell_{g}}{\rho} \frac{\mathcal{K}}{\gamma_{r}^{2}} .
$$

The gain length $\ell_{g}$ is related to dimensionless Pierce parameter $\rho$ as $\ell_{g}=1 /\left(2 k_{u} \rho\right)$, and $\rho$ reads

$$
\rho=\left[\frac{1}{16} \frac{I_{0}}{I_{\alpha}} \frac{\mathcal{K}^{2}}{\gamma_{r}^{3} \sigma_{b}^{2} k_{u}^{2}}\right]^{1 / 3},
$$

where $\sigma_{b}$ is the rms transverse size of the electron bunch, $\gamma_{r}$ is the average resonant energy in units of the rest mass, $\bar{v}_{\|}$is the average longitudinal velocity, and $I_{\alpha}=$ $m_{e} c^{3} / e<0$ and $I_{0}$ are the Alfven current and the peak bunch current, respectively. The dimensionless length of the electron bunch $\tau_{b}$ is related to physical length $L_{b}=\bar{v}_{z \mid q} T_{b}$ by

$$
\tau_{b}=L_{b} / L_{c}
$$

where $L_{c}=\lambda_{r} /(4 \pi \rho)$ is the cooperation length.

It is advantageous to explicitly express that the electric field is copropagating with electrons. To this end we define the complex field amplitude by the relation $F=$ $\operatorname{Re}\left\{\tilde{F} \mathrm{e}^{i \omega_{r}(z / c-t)}\right\}$, where $\omega_{r}=2 \omega_{u} \gamma_{r}^{2} /\left(1+\mathcal{K}^{2}\right) / 2$ is the resonant frequency that corresponds to ideal synchronism of bunches with the radiation field at the undulator entrance. Then, using the normalization (8), in the Compton limit $\rho \ll 1$ we get the dimensionless self-consistent system of equations in the form

$$
\begin{aligned}
\frac{\mathrm{d} \tau_{q}}{\mathrm{~d} \zeta}= & 2 \rho \mu_{q}, \\
\frac{\mathrm{d} \mu_{q}}{\mathrm{~d} \zeta}= & -2 \operatorname{Re}\left\{\tilde{F}\left(\zeta, \tau_{q}\right) \mathrm{e}^{i \tau_{q} /(2 \rho)}\left(1-\mathrm{e}^{i \zeta / \rho}\right)\right\}, \\
\tilde{F}(\zeta, \tau)= & \frac{1}{\bar{n}_{\|}} \sum_{q=1}^{Q_{b}} \int_{0}^{\zeta} \chi_{q} e^{-i\left[\left(\tau+\zeta^{\prime}-\zeta\right) / 2 \rho\right]} \\
& \times \delta\left[\tau+\zeta^{\prime}-\zeta-\tau_{q}\left(\zeta^{\prime}\right)\right] \mathrm{d} \zeta^{\prime},
\end{aligned}
$$

where $\bar{n}_{\|}$is the number of electrons per unit $\tau$ entering the interaction region at $\zeta=0$, and $\chi_{q}$ is the charge weighting parameter $\chi_{q}=I_{\perp}(\zeta=0, \tau) / I_{0}$. Recall that $I_{0}$ is the peak bunch current. The dimensionless energy of $q$ th electron, $\mu_{q}$, is related to its dimensional longitudinal velocity, $v_{z \mid q}$, by

$$
\mu_{q}=\frac{1-\bar{v}_{\|} / v_{z \mid q}}{2 \rho\left(1-\bar{\beta}_{\|}\right)}
$$

Note that in deriving Eq. (11) we ignored undulator harmonics since CSE at the third harmonic is about 2 orders of magnitude smaller than at the fundamental frequency (higher harmonics are even smaller) since the change in the bunch current over one period of a harmonic is smaller and hence the source term driving CSE is smaller [22]. The equation for the radiation field $\tilde{F}(\zeta, \tau)$ in our system (11) is equivalent to the corresponding one of Krinsky [10] and differs from that of McNeil et al. [11] by the term $\left(1-\mathrm{e}^{i \zeta / \rho}\right)$, which is omitted in [11] and relevant in the case of bunches comparable to or shorter than the radiation wavelength [10].

The model (11) is a 1D nonaveraged FEL model; however, the 3D effects can be taken into account by using an effective value of the FEL parameter $\rho_{\text {eff }}$ calculated with Xie's fitting formula [42,43]. Xie's formula accounts for the effects of emittance ("matched" bunch focusing is assumed in what follows), energy spread, as well as for diffraction. The latter turns out to be the most severe factor of degradation of the FEL process such that the effective 3D FEL parameter $\rho_{\text {eff }}$ is almost 2 times smaller than the 1D FEL parameter $\rho$. Since the energy spread is accounted for by $\rho_{\text {eff }}$, only several thousands of macroparticles are used in simulations.

To perform a vast amount of calculations required for obtaining statistically valid results, we developed an unconditionally stable and fast mathematical algorithm to solve Eqs. (11). Our mathematical algorithm is conceptually similar to that of the code "FAST" [44] and does not impose constraints on discretization steps in longitudinal coordinate, observation time, and entrance time of electrons. The numerical implementation is given in Appendix A. The field is calculated on a mesh surrounding the bunch and then it is interpolated to the electron's positions, thus decreasing the computational time as compared to finite-difference methods that uses a mesh dependent on boundary conditions (advantages of integral methods over differential ones for injector design codes are discussed in [45] in detail). Our simulation code was verified against the analytical result (6) in a low-gain regime and against results presented in [11] for a deep nonlinear regime, and excellent agreement was found.

\section{SELF-AMPLIFIED COHERENT SPONTANEOUS EMISSION}

The coherent spontaneous emission originates from the gradient of the beam current and reaches its maximal value for the current distribution of a rectangular shape. The later is commonly used in simulations and we will also start our analysis with such an approximation. Although the rectangular current distribution is an idealized case it makes the result more universal. The impact of a finite rise time of the front of a current pulse can be estimated for a desired bunch current gradient using our calculations of the characteristics of self-amplified CSE for different rise times of the current pulse. To model shot noise we use the technique proposed in [46] that creates an ensemble of macroparticles with the same statistical proprieties as the real electron ensemble. The 
TABLE I. SwissFEL injector parameters after bunch compression.

\begin{tabular}{lll}
\hline \hline Parameter & Symbol & Value \\
\hline Electron energy & $\gamma_{r} m_{e} c^{2}$ & $250 \mathrm{MeV}$ \\
Bunch peak current & $I_{0}$ & $350 \mathrm{~A}$ \\
Transverse rms size & $\sigma_{b}$ & $55 \mu \mathrm{m}$ \\
Energy spread & $\sigma_{\gamma} m_{e} c^{2}$ & $64 \mathrm{keV}$ \\
Normalized emittance & $\varepsilon_{n}$ & $0.36 \mathrm{~mm} \mathrm{mrad}$ \\
Bunch duration & $T_{b}$ & $190 \mathrm{fsec}$ \\
\hline \hline
\end{tabular}

main parameters of the SwissFEL injector and the proposed FEL are listed in Tables I and II [47].

To identify different regimes of the FEL we have calculated the peak and average power as a function of the interaction distance along the undulator. The results are shown in Fig. 3. The peak power is mainly dictated by CSE whereas the average power strongly depends on shot noise. It is interesting to note that the distribution of the average power is visually almost identical to the LCLS power distribution calculated using GENESIS; cf. Fig. 3 of our work and Fig. 8 of Ref. [43]. From Fig. 3 one can see that the low-gain regime extents to around $30 \lambda_{u}$, the high-gain interaction occurs approximately in the region from $30 \lambda_{u}$ to $80 \lambda_{u}$, and the FEL saturates beyond this region.

As we found in the previous section, interference of elementary electromagnetic impulses emitted by uniformly distributed electrons moving with constant velocities results in a series of radiation pulses that are one cycle in duration. If we take into account the reaction of the field on the electrons dynamics, then the result found earlier remains correct in the low-gain regime, as it is shown in the top left plot in Fig. 4. In this figure we present the radiation power as a function of time at different longitudinal positions $z=N_{u} \lambda_{u}$ in the undulator for a FEL with and without shot noise in the electron bunch. Initially, the radiated field is small and synchronous with the electrons and its reaction accumulates as the bunch moves through the interaction region such that the high-gain regime starts to

TABLE II. Main parameters of the FEL.

\begin{tabular}{lcl}
\hline \hline Parameter & Symbol & Value \\
\hline Undulator period & $\lambda_{u}$ & $4 \mathrm{~cm}$ \\
Undulator parameter & $\mathcal{K}$ & 3.2 \\
Number of undulator periods & $N_{u}$ & 200 \\
FEL wavelength & $\lambda_{r}$ & $0.511 \mu \mathrm{m}$ \\
FEL parameter & $\rho$ & 0.0095 \\
Cooperation length & $L_{c}$ & $4.29 \mu \mathrm{m}$ \\
Gain length & $\ell_{g}$ & $33.57 \mathrm{~cm}$ \\
Normalized bunch length & $\tau_{b}$ & 13.3 \\
Effective number of electrons & $\bar{n}_{\|}$ & $1.25 \times 10^{9}$ \\
Effective FEL parameter & $\rho_{\mathrm{ef}}$ & 0.0053 \\
\hline \hline
\end{tabular}

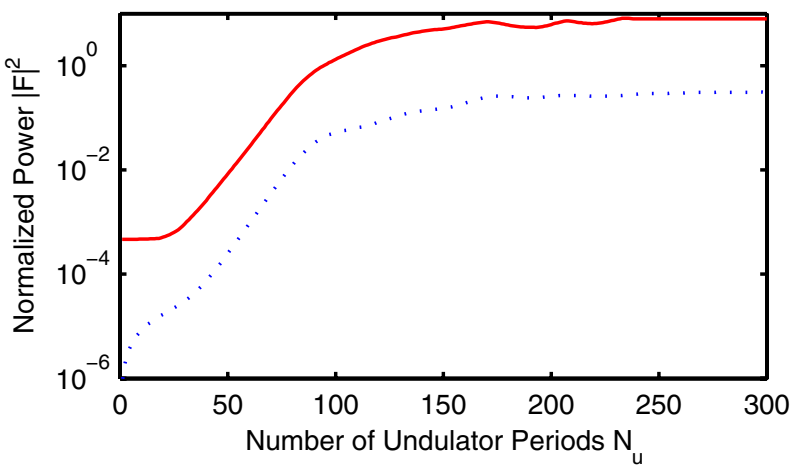

FIG. 3. The peak (solid red curve) and average (dotted blue curve) power as a function of the undulator distance.

dominate and the field amplitude increases as we can see on the plots for $N_{u}=35$ and $N_{u}=45$ in Fig. 4. Later, the radiated field changes the electron trajectories such that the slippage time, which depends on the velocity of electrons, becomes different for different electrons. In turn, the temporal structure of the radiation field from accelerated electrons becomes more complicated such that simple
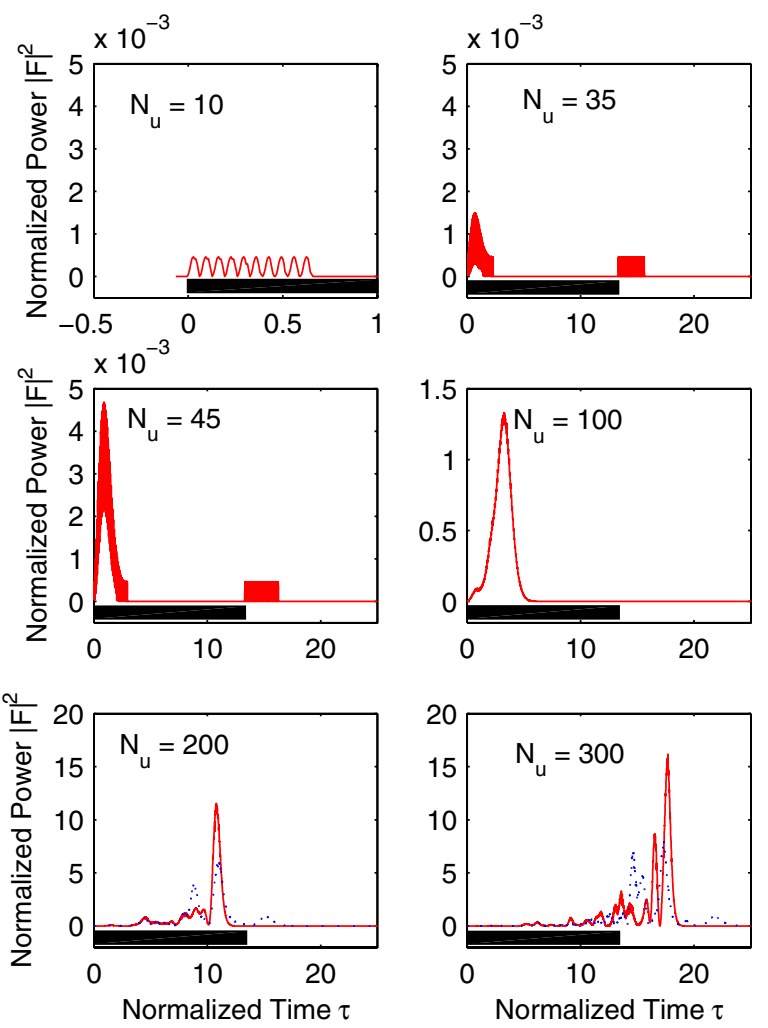

FIG. 4. Normalized power vs normalized time at different longitudinal positions in the undulator. The solid red curves depict the results for simulations without shot noise whereas the dotted blues curves depict the simulations that take into account shot noise. The arrival time of electrons is shown by the black boxes lying under the abscissas. Note that there are different scales on the plots. 
interference does not take place any more and the selfamplification of CSE occurs.

A series of EM pulses results in the single wide pulse at the end of the high-gain regime (plot for $N_{u}=100$ in Fig. 4) because the electron bunch in the undulator is an active medium with some dispersion of the group velocity such that widening of electromagnetic pulses occurs during their propagation through the medium. The effect of the electron bunch dispersion is demonstrated in [44] where it is shown that in the linear high-gain regime the group velocity is $v_{\mathrm{gr}}=c\left(1-1 / 3 \bar{\gamma}_{\|}^{2}\right)$ and the relative slippage of the wave packets (SASE spikes) with respect to the electron beam is three time less than the kinematic slippage. In the nonlinear regime the group velocity of the wave packets is close to the velocity of light.

In the nonlinear regime (plots for $N_{u}=200$ and $N_{u}=$ 300 in Fig. 4) the radiation pulse has a clear spike with a duration of the order of the cooperation length, $\Delta \tau \sim 1$ $\left(\Delta t \sim L_{c} / \bar{v}_{\|}\right)$. This main spike overtakes the bunch and leaves it behind approximately at the longitudinal position $\zeta \sim \tau_{e}+1\left(z \sim \bar{v}_{\|} T_{b}+L_{c}\right)$ and then it propagates without distortions in free space. This corresponds to $N_{u}=300$ in the plotted simulation. Whereas shot noise has a negligible effect on the radiation pulse shape at low gain, in the high-gain regime it results in a substantial distortion of the radiation pulse and reduction of the emitted power in the nonlinear regime. In the high-gain regime small random distortions are amplified in an exponential fashion to a noticeable level.

To mitigate the shot noise effect several techniques have been proposed, and FELs with a reduced level of shot noise are under active study $[18,20,21]$. At the same time, to the authors knowledge only a linear theory of such FELs have been published. The problem regarding to what extent the bunch noise has to be suppressed in order to obtain welldetermined radiation pulses requires further studies, and we address this question in our simulations of stimulated CSE.

To characterize the level of noise at wave number $k$, we will follow Ref. [14] and define the noise factor as

$$
\Gamma(k, z)=\frac{1}{Q_{b}} \sum_{q, p} e^{i k\left[s_{q}(z)-s_{p}(z)\right]},
$$

where $s_{q}(z)$ is the longitudinal bunch coordinate of particle $q$ at position $z$ in the undulator and $k=2 \pi / \lambda$ is the wave number. One can check that if the particle positions are uncorrelated, this results in $\langle\Gamma(k, z)\rangle=1$, where $\langle\ldots\rangle$ stands for the statistical averaging. The situation $\langle\Gamma(k, z)\rangle<1$ corresponds to the case of anticorrelated (quiet) bunches. If the bunch energy spread is small, then magnetic compression causes microbunching instabilities such that $\langle\Gamma(k, z)\rangle \sim G$, where $G$ is the microbunching gain. This situation corresponds to the correlated bunch case. In what follows, we will consider not only quiet but also correlated bunches since even in the presence of a laser heater the microbunching gain can greatly exceed unity at a certain wavelength when a multistage compression system is used. In order to study the effect of noise on FEL performance, we have calculated the probability density distribution of the maximum power in the radiation pulse for different values of the noise factor $\langle\Gamma(k)\rangle$; see Fig. 5. Recall that the normal level of shot noise corresponds to $\langle\Gamma(k)\rangle=1$. It turns out that just as for the SASE FEL the envelope of the probability density distribution of the self-amplified CSE pulses can be accurately approximated by the Gamma distribution with an appropriate value of the $M$ parameter [48] as it is shown in the same Fig. 5. The $M$ parameter is defined as $1 / \sigma^{2}\left(\left|F_{\max }\right|^{2}\right)$, where $\sigma^{2}\left(|F|^{2}\right)=\left(|F|^{2}-\left\langle|F|^{2}\right\rangle\right)^{2} /\left\langle|F|^{2}\right\rangle^{2}$ is the relative dispersion of the normalized power of radiation pulses. For $\langle\Gamma(k)\rangle \ll 1$ the $M$ parameter increases in a quasilinear way as is demonstrated in Fig. 6.

As one can see in Fig. 7 noise reduction is accompanied by an increase in the mean maximum power $\mathrm{E}\left(\left|F_{\max }\right|^{2}\right)$ and by a decrease in the rms width $\sigma\left(\left|F_{\max }\right|^{2}\right)$ of distribution function of the normalized maximum power. The latter directly determines the FEL coherence because $\sigma\left(|F|^{2}\right)$ is related to the second-order correlation function of zero argument, $g_{2}(0)$, by [44]
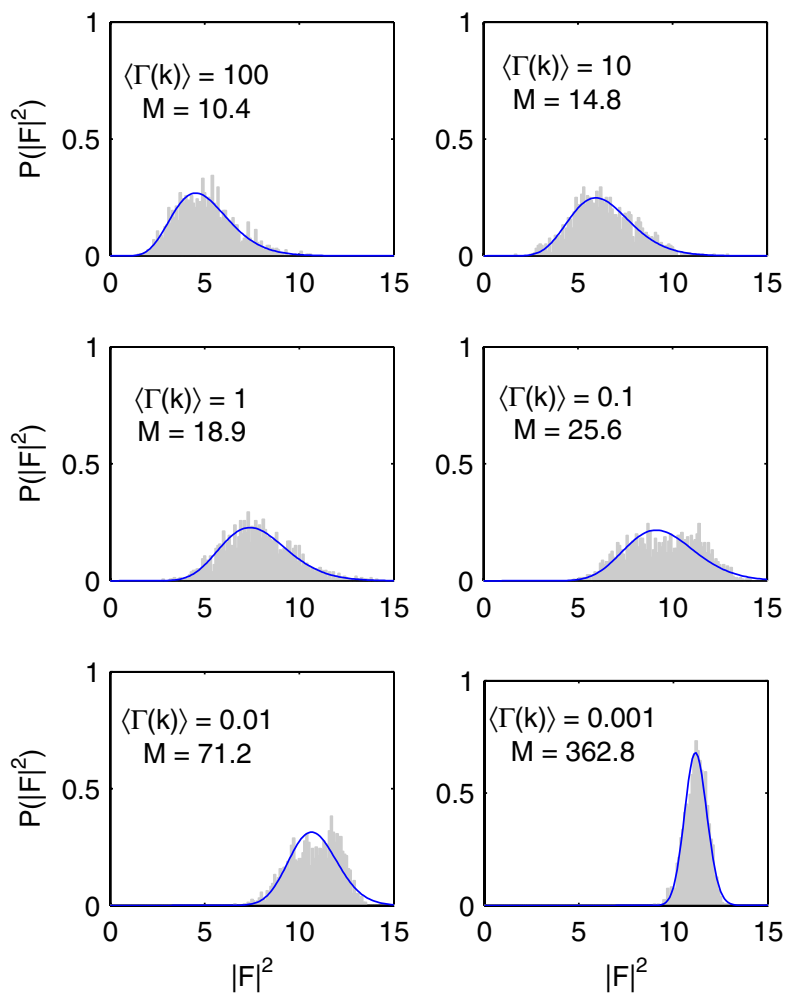

FIG. 5. Histograms of the probability density distribution, $p(|F|)^{2}$, of the normalized maximal power $|F|^{2}$ for different levels of noise. The solid blue curves represent corresponding Gamma distributions. Calculations have been performed with 1600 statistically independent simulations. The bunch and FEL parameters are given in Tables I and II. 


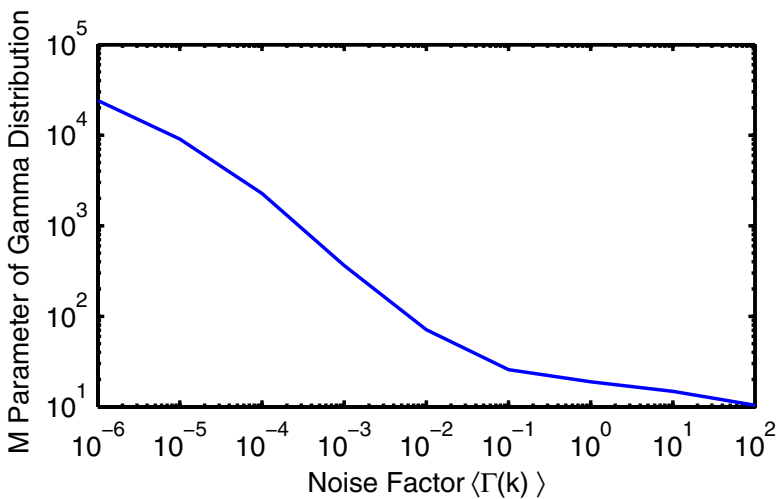

FIG. 6. $M$ parameter as a function of the noise factor. Both axes are in a logarithmic scale.

$$
g_{2}(0)=1+\sigma\left(|F|^{2}\right) .
$$

It follows from the theory of statistical optics that the cases of $g_{2}(0)=1$ and $g_{2}(0)=2$ correspond to stabilized single-mode laser radiation and to completely chaotic radiation from a thermal source, respectively. As the noise factor decreases $g_{2}(0)$ tends to unity. For example, given $\langle\Gamma(k)\rangle=10^{-3}$ the normalized rms deviation of the maximal power is $5.25 \%$ and $g_{2}(0) \approx 1.003$. Thus, quiet bunches produce near single-mode pulses.

Noise with the level corresponding to the normal shot noise level almost halves the mean maximum power as compared to the case of a zero noise level (7.8 of $\left|F_{\max }\right|^{2}$ against 11.7 of $\left|F_{\max }\right|^{2}$ ). In the latter case the maximum physical power is around $5.5 \mathrm{GW}$ and the energy stored in the main radiation spike is around $100 \mu \mathrm{J}$. The normalized rms power width and the second-order correlation function of zero argument are $23 \%$ and 1.053 , respectively. It turns out that for $\langle\Gamma(k)\rangle \ll 1$ the logarithm of the normalized rms width, $\sigma\left(\left|F_{\max }\right|^{2}\right)$, shows almost linear dependence on $\langle\Gamma(k)\rangle$ so that $\sigma\left(\left|F_{\max }\right|^{2}\right)$ is proportional to $\langle\Gamma(k)\rangle^{1 / 3}$. One can see that $\sigma\left(\left|F_{\max }\right|^{2}\right)$ diminishes slowly as $\langle\Gamma(k)\rangle$ decreases and the reduction of the noise level by several orders of magnitude is required to obtain the normalized rms width of the maximum power less than $10 \%$.

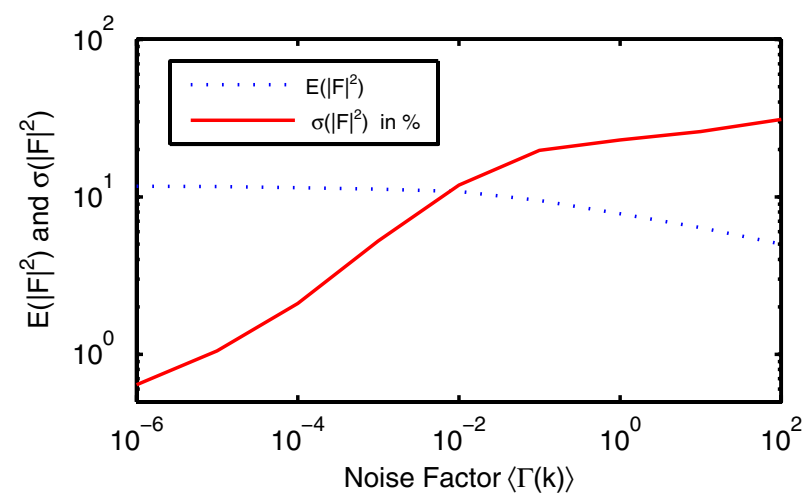

FIG. 7. The mean and standard deviation of $|F|^{2}$ versus noise factor. Both axes are in a logarithmic scale.

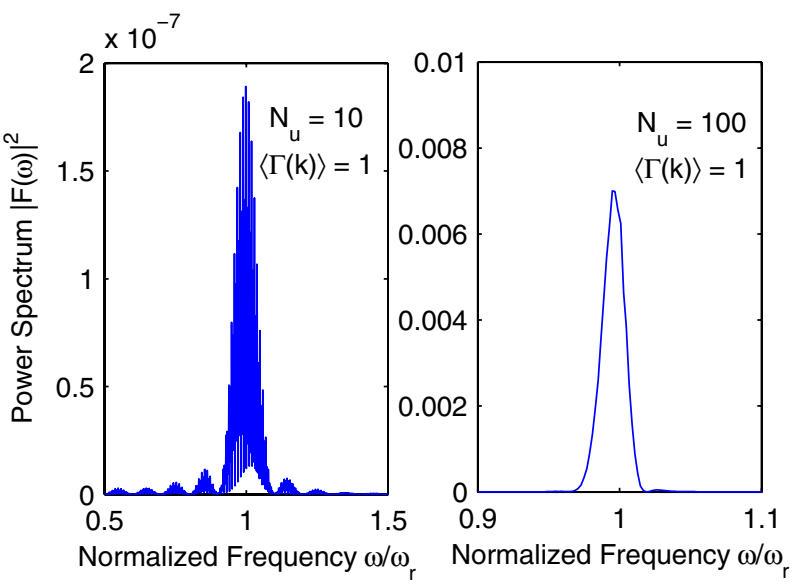

FIG. 8. Typical spectrums of the dimensionless power $|F(\omega)|^{2}$ vs the frequency normalized to the resonant frequency $\omega_{r}$.

Typical spectra of the dimensionless power $|F(\omega)|^{2}$ created by the bunch with a normal level of shot noise are shown in Fig. 8. The radiation at the beginning of the nonlinear regime $N_{u}=100$ is defined by the narrow-band fraction of the initial broadband radiation at the FEL startup $N_{u}=10$. It turns out that the FEL spectrum at the undulator output $N_{u}=200$ shown in Fig. 9 is almost independent on the noise level and is mainly dictated by the properties of the self-amplified CSE process. The side band frequencies are related to the synchrotron oscillations of electrons in the nonlinear regime.

The Fourier transform limited pulses are important in many applications, and it turns out that a FEL based on CSE is able to produce such pulses. We found that the timebandwidth product (defined in this paper as a product of the pulse rms width in the time domain, $\sigma_{f}$, and the pulse rms width in the frequency domain, $\sigma_{t}$ ) depends weakly on the noise level and is close to unity in the nonlinear regime; see Fig. 10. We also found that the time-bandwidth product is
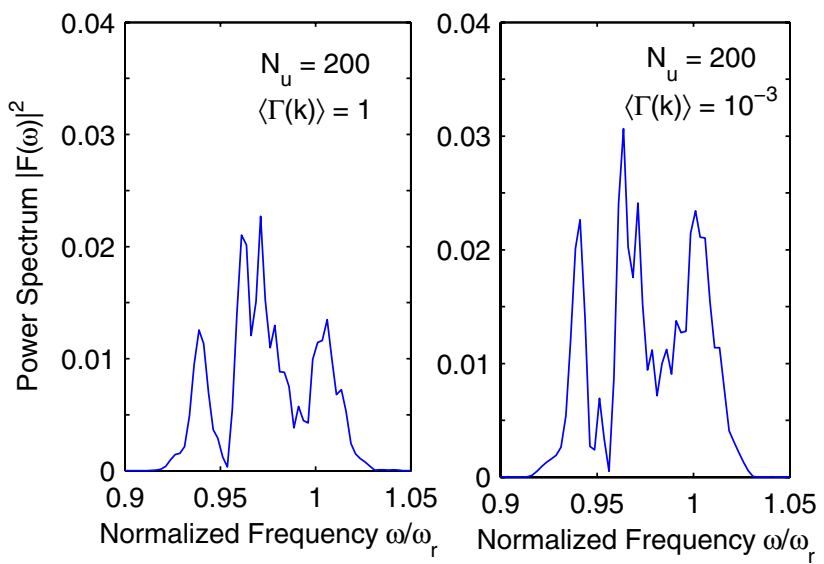

FIG. 9. Spectrum of the dimensionless power $|F(\omega)|^{2}$ vs the frequency normalized to the resonant frequency $\omega_{r}$. The results are averaged over 1600 statistically independent simulations. 


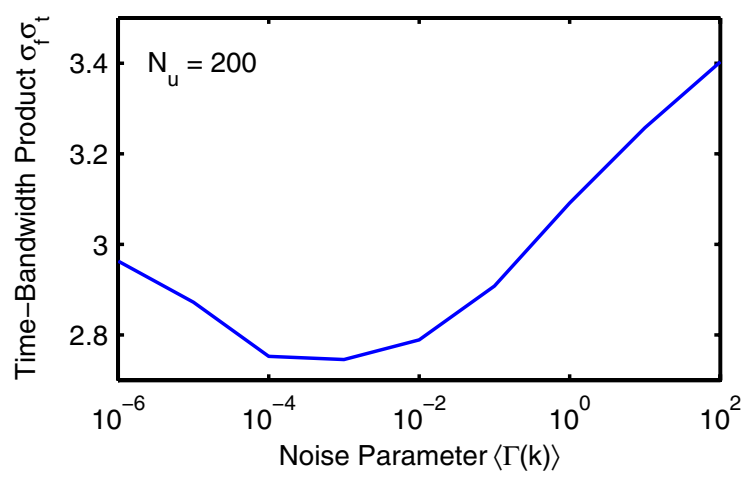

FIG. 10. The time-bandwidth product vs noise parameter. The result is statistically averaged.

mainly determined by the bunch length and that it strongly depends on the interaction distance. It attaints its minimal value approximately at the beginning of the nonlinear stage of the bunch-wave interaction as one can see in Fig. 11.

Up to now we have considered the FEL with a rectangular current pulse such that the current gradient at the bunch tail and head is infinite. Let us briefly discuss how the results will change if the rise time is finite and the gradient is limited. Let $s$ be the dimensional coordinate along the bunch with $s=0$ corresponding to the bunch tail. Then, the current pulse profile of interest reads

$$
\frac{I(s)}{I_{0}}= \begin{cases}\sin ^{2}\left[\frac{\pi}{2} \frac{s}{\Delta \lambda}\right] & s \leq \Delta \lambda, \\ 1 & \Delta \lambda<s \leq L_{b}-\Delta \lambda, \\ \cos ^{2}\left[\frac{\pi}{2} \frac{s-L_{b}-\Delta \lambda}{\Delta \lambda}\right] & L_{b}-\Delta \lambda<s \leq L_{b}, \\ 0 & s>L_{b}\end{cases}
$$

and is schematically shown in Fig. 12. We performed simulations with different current rise times $\Delta \lambda / c$ for a reduced level of shot noise. One can see in Fig. 13 the mean of $\left|F_{\max }\right|^{2}$ quickly decreases as $\Delta \lambda$ increases but the normalized rms deviation of $\left|F_{\max }\right|^{2}$ is almost independent of the bunch current gradient. This result is qualitatively similar to that for the normal level of shot noise. The latter result is nontrivial but has a straightforward explanation.



FIG. 11. The time-bandwidth product vs undulator distance. There is no shot noise in the simulations.

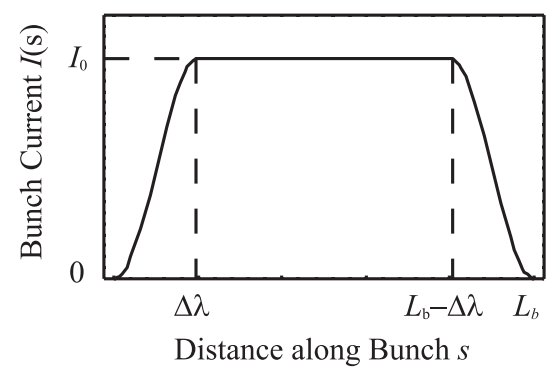

FIG. 12. The bunch current at the undulator entrance as a function of the distance along the bunch.

The level of fluctuations of the radiation field depends not only on the electron noise but also on the FEL gain and the interaction distance. For the given parameters the gain is determined by CSE, and as the gradient is reduced the FEL gain is reduced as well such that field fluctuations are enhanced less in a given length. Indeed, we already found at the beginning of this section that the impact of shot noise on the radiation field profile becomes important only in the nonlinear regime as is shown in Figs. 3 and 4. We conclude that the reduced level of power fluctuation is preserved as the current gradient is reduced such that we expect that the results obtained for the rectangular current pulse are qualitatively correct even for a bunch current pulse with a rise time corresponding to several FEL wavelengths.

CSE is typically associated with long wavelength FELs. However, this fact is not related to fundamental FEL limitations on the generation of stimulated CSE but to technological challenges in the formation of bunches capable to effectively drive CSE at short wavelengths. Our estimates for the FEL based on CSE with a finite current gradient and reduced shot noise presented in the Appendix B show that such FELs may be effective sources in the VUV region. Specifically, the FEL process starts from spontaneous undulator emission and the ratio of the power of CSE to the power of incoherent emission is $Q_{b}|\mathcal{F}(\omega)|^{2} / g[\langle\Gamma(\omega)\rangle]$, where $\mathcal{F}(\omega)$ is the form factor of the bunch [9], and $g[\langle\Gamma(\omega)\rangle]$ takes into account the

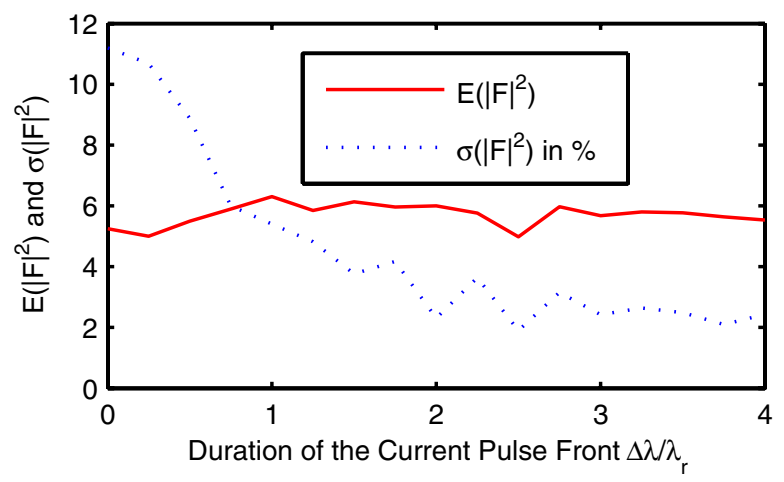

FIG. 13. Mean and normalized rms deviation of $\left|F_{\max }\right|^{2}$ vs length of the current pulse front. The noise level is 3 orders of magnitude below the normal shot noise level, $\langle\Gamma(k)\rangle=10^{-3}$. 
reduction of incoherent emission due to the shot noise reduction [18]. Therefore, if the incoherent emission is reduced, then CSE can be extended to shorter wavelengths. We found that VUV FELs based on self-amplified CSE require the reduction of shot noise by 3-4 orders of magnitude in order to have radiation pulses with a welldetermined temporal structure.

\section{SHOT NOISE SUPPRESSION AND CONTROLLED MICROBUNCHING INSTABILITY}

In this section we propose and subsequently analyze feasibility of a scheme that allows for the formation of quiet electron bunches with a high current gradient at the bunch tail. We will consider the evolution of an electron bunch from the injector to the undulator passing through a laser heater, a bunch compressor, and a shot noise suppression section [49] as it is shown in Fig. 14. While the previous section describes the FEL performance in a rigorous way within a $1 \mathrm{D}$ approximation, in this section we will give order-of-magnitude estimates of the relevant bunch characteristics because our intention is to reveal the physics of the process rather than to present detailed technical specifications. Let us consider the bunch evolution in each part of the system.

\section{A. Controlled microbunching instability}

Similar to what was done in Ref. [38] we neglect the microbunching process and energy modulation accumulated inside the electron injector and analyze the evolution of the electron density modulation starting from the linear accelerator of the SwissFEL [47]; see the schematic Fig. 14. In fact, in the early accelerating stage at the optical region $\lambda_{D} \gg \lambda$, where $\lambda_{D}$ is the Debye wavelength, the microbunching process is washed out by Landau damping (see discussion in [17]). Recent experiments [17] confirm that at the injector exit there is no noticeable collective microbunching or current-velocity noise correlation. Therefore, we assume that at the injector exit, electron bunches have an uncorrelated energy spread with a Gaussian distribution characterized by $\sigma_{\gamma_{0}} m_{e} c^{2}=3 \mathrm{keV}$ and a density modulation given by a bunching factor

$$
b_{0}(k)=\frac{1}{Q_{b} e c} \int I(s) \mathrm{e}^{-i k s} d s
$$

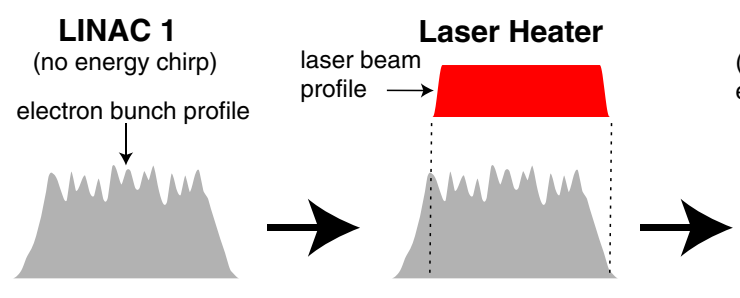

which is related to the noise factor $\Gamma(k)$ by $\langle\Gamma(k)\rangle=$ $Q_{b}|b(k)|^{2}$. Here, $I(s)$ is the bunch current and $s$ is the longitudinal coordinate along the bunch. The microbunching gain $G(k)$ is defined as a ratio of the bunching factors after and before compression. Because of the inhomogeneity of the electron density, the space-charge oscillations are initiated and the conversion of density modulations into energy modulations as well as the reconversion occur each quarter plasma oscillation period after the injector.

Note that dependent on specific injector parameters the electron density modulation (16) may be caused not only by shot noise but also by intensity modulations of the photocathode drive laser. Specifically, the existence of the microbunching instability at the thermionic gun of the Spring-8 Angstrom Compact Free Electron Laser (SACLA) has confirmed that microbunching instability can come from the shot noise nature of the bunch. At the same time, experimental studies of the origin of the microbunching instability in the FEL injector of the National Synchrotron Light Source Development Laboratory (SDL) [50] show that the only significant cause of the microbunching instability under normal operational conditions is the longitudinal modulation of the photocathode laser pulse. The intensity modulation of the photocathode drive laser also plays a significant role at the SwissFEL injector [51].

Because of impedances (mainly the space-charge impedance), any electron bunch density modulation gives rise to the energy modulation as the bunch propagates through a drift section. This energy modulation is converted into an additional density modulation during magnetic compression, the so-called microbunching gain. The microbunching instability is very sensitive to the uncorrelated energy spread of the electron beam; increasing it within the FEL tolerance provides strong Landau damping to counteract the instability. To this end Saldin et al. [37] suggested a laser heater that makes use of a resonant beam laser interaction in a short undulator to induce rapid energy modulation at optical frequencies to increase effective energy spread.

Let us consider the microbunching process from the injector end to the output of the bunch compressor with and without the laser heater. For our analysis of the microbunching gain we use the analytical model developed in [38]. The laser spot size is assumed to be matched to the transverse size of the electron bunch so that the heating is

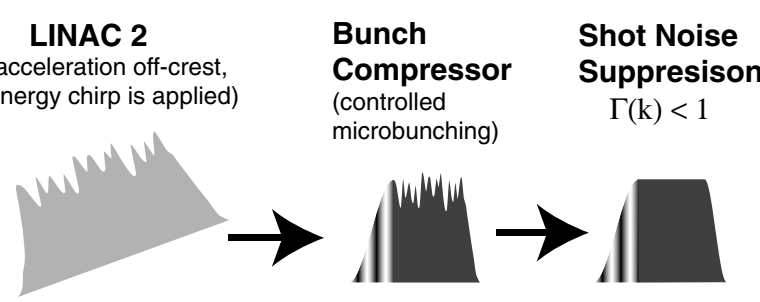

FIG. 14. The schematic illustration of a possible formation of the quiet bunch with a high current gradient at the bunch tail. 


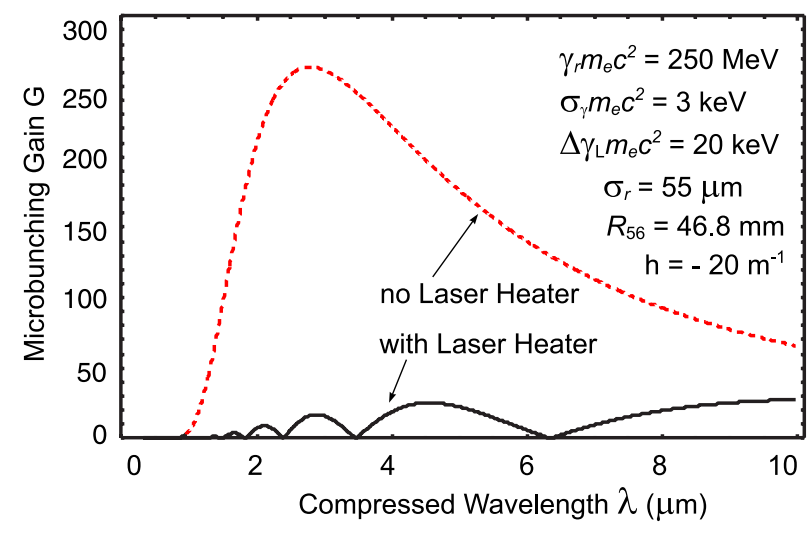

FIG. 15. Microbunching gain vs wavelength after compression with and without taking into account the effect of the laser heater.

more uniform in terms of the energy spread. The effect of coherent synchrotron radiation in a bunch compressor is calculated using formulas from [52]. The details on the LINAC configuration and the bunch acceleration can be found in [47].

In Fig. 15 we present the results of calculations of the microbunching gain for the system with and without a laser heater. Without heating of electrons, an appreciable microbunching gain is observed approximately at a compressed wavelength of $2.7 \mu \mathrm{m}$. Recall that the chicane compresses simultaneously the bunch length and the modulation wavelength by the compression factor $C=\left(1+h R_{56}^{(c)}\right)^{-1}$, where $R_{56}^{(c)}=46.8 \mathrm{~mm}$ is the chicane dispersive strength and $h=-20 \mathrm{~m}^{-1}$ is the energy chirp. For the considered set of parameters $C \approx 16$ so that the maximum microbunching occurs at an initial modulation wavelength of around $42 \mu \mathrm{m}$. The microbunching strongly depends on the uncorrelated energy spread [34], and for the wavelengths shorter than the cutoff wavelength $\lambda_{c}=$ $2 \pi R_{56}^{(c)} \sigma_{\delta_{0}} /\left(1+h R_{56}^{(c)}\right) \approx 5.5 \mu \mathrm{m}$ it is smeared out. Here, $\sigma_{\delta_{0}}=\sigma_{\gamma_{0}} / \gamma=1.2 \times 10^{-5}$ is the relative incoherent energy spread just before compression. An increase of the incoherent energy spread to $10.8 \mathrm{keV}$ by means of a laser heater results in strong suppression of the microbunching gain as it is shown in 15. The maximum energy modulation, $\Delta \gamma_{L} m_{e} c^{2}=20 \mathrm{keV}$, produced by the laser heater, is chosen in such a way that it results in an effective microbunching suppression on the one hand, and it does not affect substantially the performance of the shot noise suppression downstream of the bunch compressor. Recall that the bunch compressor also increases an incoherent energy spread by the compression factor $C$ so that the energy spread of the heated bunch after compression is around $\sigma_{\gamma} m_{e} c^{2}=170 \mathrm{keV}$.

Therefore, if we modulate the bunch tail with a period of around $40 \mu \mathrm{m}$ and use the laser heater to heat only the core of the bunch leaving the tail unheated, as it is shown in
Fig. 14, then we obtain a microbunched tail and a steep variation of the electron density at the tail of the bunch core. The modulation can be realized by means of the photocathode laser or using a laser modulator just upstream of the bunch compressor. Note that the microbunching gain in the vicinity of the resonant wavelength, which is $0.511 \mu \mathrm{m}$, is small even without a laser heater but the latter is needed to be sure that the normal level of shot noise is preserved during compression. Otherwise a subsequent reduction of shot noise might be less efficient.

\section{B. Shot noise suppression}

A reduction of shot noise downstream of the bunch compressor can be achieved by employing either the scheme proposed by Gover and Dyunin [12] or the scheme proposed by Ratner et al. [14]. The first scheme uses only a drift channel of an appropriate length whereas the second one employs a particle-to-particle interaction region (for example a drift channel) and a chicane. The latter allows obtaining stronger noise suppression for the same drift length compared to Gover's scheme or weaker noise suppression but with a shorter drift channel. Specifically, Gover's scheme works only if the drift channel length, $L_{d}$, is around a quarter of the plasma wavelength $L_{d} \approx \lambda_{p} / 4$. In that case the shot noise suppression factor reads [12]

$$
\langle\Gamma(k)\rangle=\frac{\sigma_{\gamma} m_{e} c^{2} / e}{4 \pi I_{0} / c} k \theta_{p r} S_{b},
$$

where $\theta_{p r}=r_{p} \omega_{p} / \bar{v}_{z}$ and $\omega_{p}=\left(4 \pi n_{e} e^{2} / m_{e} \gamma^{3}\right)^{1 / 2}$ are the longitudinal plasma wave number and the relativistic plasma frequency, respectively; $\bar{v}_{z}$ and $\gamma$ are the average longitudinal bunch velocity and the bunch energy in terms of the rest mass, respectively; and $n_{e}$ is the plasma density. The plasma reduction factor

$$
r_{p}=1-2 I_{1}\left(\sigma_{b} k / \gamma\right) K_{1}\left(\sigma_{b} k / \gamma\right)
$$

takes into account a finite transverse size of the electron bunch. Here, $I_{1}(x)$ and $K_{1}(x)$ are modified Bessel functions. The result (17) is correct when the transverse extent of the longitudinal Coulomb field of each electron is wider than the bunch diameter [12], i.e.

$$
\gamma \lambda \gg 2 \sigma_{b} \text {. }
$$

Then, all electrons in the bunch cross section create the in-phase longitudinal Coulomb field. One can see that the maximal noise suppression in Gover's scheme (17) is limited by the uncorrelated energy spread $\sigma_{\gamma}$. Note that the shot noise reduction is accompanied by the spectral energy noise enhancement such that the spectral energy noise factor is equal to $1 /\langle\Gamma(k)\rangle$.

In Ratner's scheme the noise reduction occurs in a chicane, where the energy modulation gained in the particle-to-particle interaction region is translated into a 
homogenization of the bunch density. The noise factor reads

$$
\begin{aligned}
\langle\Gamma(k)\rangle= & 1-2\left(Q_{b} / L_{b}\right) k R_{56} \operatorname{Im}[\tilde{h}(k)] e^{-k^{2} R_{56}^{2} \sigma_{\delta}^{2}} \\
& +\left(Q_{b} / L_{b}\right)^{2} k^{2} R_{56}^{2}|\tilde{h}(k)|^{2} e^{-k^{2} R_{56}^{2} \sigma_{\delta}^{2}},
\end{aligned}
$$

where $\sigma_{\delta}=\sigma_{\gamma} / \gamma$ is the normalized energy spread, and $R_{56}$ is the dispersive strength of the chicane. Equation (20) is applicable only if $Q_{b} k^{2} R_{56}^{2} \ll 1$ so that the effect of a large energy spread cannot be considered. If the drift channel is used as an interaction region, then the energy modulation, $\tilde{h}(k)$, is

$$
\tilde{h}(k)=\frac{i 4 \pi r_{e} L_{d} r_{p}}{k S_{b} \gamma},
$$

where $r_{e}$ is the classical electron radius. The maximum noise suppression is also limited by the energy spread as in Gover's scheme. At the same time, in Ratner's scheme the noise factor can be regulated by changing the chicane dispersive strength $R_{56}$ (the optimal value of $R_{56}$ is around $\left.1 / k\left(Q_{b} / L_{b}\right) \operatorname{Im}[\tilde{h}(k)]\right)$ whereas in Gover's scheme it is set by the bunch parameters. Therefore, Ratner's scheme is more flexible and allows for some optimization of $L_{d}$ and $R_{56}$ to obtain a compromise between the shot noise suppression and the energy noise enhancement. The latter can be estimated using the formulas from Ref. [12].

There is no enhancement of the microbunching of the heated part of electron bunches so that the normal level of shot noise is preserved during the bunch compression, and we can apply Eqs. (17) and (20) to estimate noise reduction downstream of the bunch compressor. The noise factor for Gover's and Ratner's schemes at the wavelength region from $\lambda_{r} / 2$ to $3 \lambda_{r} / 2$ is presented in Fig. 16. Recall that $\lambda_{r}$ is the FEL resonant wavelength.

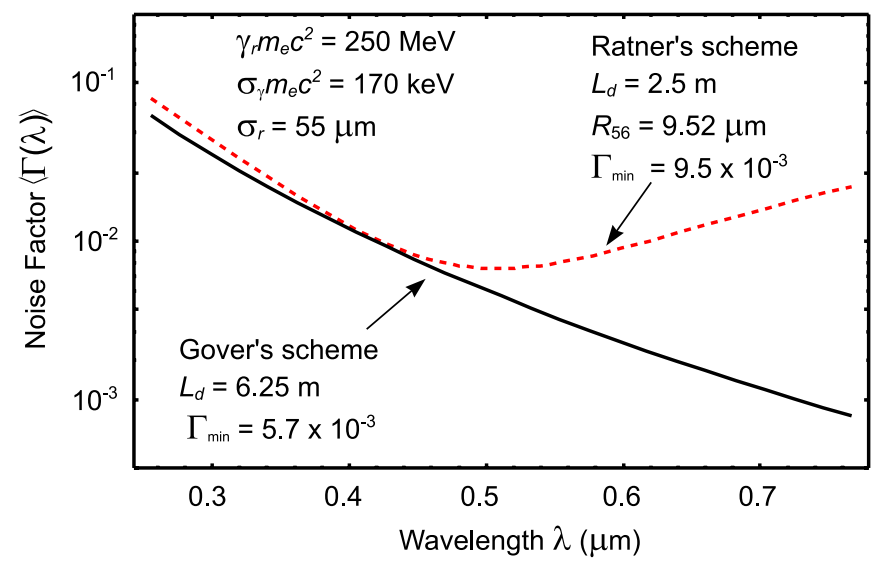

FIG. 16. Noise factor vs wavelength for two noise suppression schemes. The black solid line stands for the noise factor for the scheme employing only a drift channel (Gover's scheme) whereas the red dotted curve stand for the noise factor for the scheme employing a drift channel and a chicane (Ratner's scheme).
The relativistic plasma wavelength $\lambda_{p}$ is around $25 \mathrm{~m}$ so that the required interaction length in Gover's scheme is around $6.25 \mathrm{~m}$ and the maximum noise reduction is around $10^{2}$. One can see from Fig. 16 that the same noise reduction is achieved in Ratner's scheme with a shorter drift length of $2.5 \mathrm{~m}$. Because of the finite transverse size of electron bunches, the noise factor (20) strongly depends on the wavelength, and we chose dispersive strength $R_{56}$ to minimize $\langle\Gamma(k)\rangle$ at $\lambda=\lambda_{r}$ whereas for other wavelengths $R_{56}$ is not optimal. At the same time the noise reduction is quite uniform around the desired frequency as is demonstrated in Fig. 16 (dotted red curve) because the first derivative of $\langle\Gamma(k)\rangle$ with respect to $\lambda$ is equal to zero. Thus, the shot noise suppression section based on Ratner's scheme with a relatively short drift channel proposes a good compromise between the noise suppression effectiveness and system size. Note that Ratner's theory does not take into account the noise reduction due to the plasma oscillations in a drift channel so that Gover's and Ratner's scheme cannot be compared for the same length of a drift channel. The bunching factors at the end of the bunch compressor and noise suppression section, $b_{0}(k)$ and $b_{s}(k)$, are related by $\left|b_{s}(k)\right|^{2}=\langle\Gamma(k)\rangle\left|b_{0}(k)\right|^{2}$. Note that the considered set of parameters represents the SwissFEL accelerator and is not optimal for the proposed scheme of formation of "quiet" bunches with a high current gradient at the bunch tail. In principle, further reduction of shot noise is possible by optimizing the parameters of the accelerator, the laser heater, and the bunch compressor.

\section{DISCUSSION AND CONCLUSION}

We studied self-amplified coherent spontaneous emission in a planar FEL driven by electron bunches with different levels of density fluctuations. These density fluctuations originating from shot noise compete with an intrinsic coherent seeding driven by the current gradient and are in the focus of our investigation. Simulations are mainly performed for electron bunches with a rectangular shape of the charge density since this makes the results more universal. We found that the temporal structure of electromagnetic pulses in the studied FEL is mainly determined by the self-amplified coherent spontaneous emission because the coherent part of spontaneous undulator radiation driven by the current gradient is dominant over the incoherent one originating from shot noise. However, the incoherent emission being small leads to essential distortions of radiation pulses during amplification. To study the effect of noise on the FEL performance, we have calculated the probability density distribution of the maximal power of the radiation pulses for different values of noise factor $\langle\Gamma(k)\rangle$. It turns out that likewise to the SASE FEL the envelope of the probability density distribution of the self-amplified CSE pulses can be accurately approximated by the Gamma distribution. The rms width of the 
probability density distribution determines the temporal coherence of the FEL. We found that the coherence quickly increases as the noise level decreases and at the noise level, for example, 3 orders of magnitude below the normal shot noise level, the FEL radiation is almost completely coherent.

It turns out that the FEL based on CSE produces pulses that are almost Fourier transform limited. We found that the time-bandwidth product depends weakly on the noise level and is close to unity in the nonlinear regime. The analysis indicates that the time-bandwidth product is mainly determined by the bunch length and interaction distance, and it attaints its minimal value approximately at the beginning of the nonlinear stage of the bunch-wave interaction.

To estimate the effect of a finite electron current gradient, we have also performed calculations of the FEL characteristics for different rise times of the current pulse. We found that a reduced level of the power fluctuations are preserved as the current gradient is reduced so that we expect the results obtained for the rectangular current pulse to be qualitatively correct for a bunch current pulse with a rise time of up to several FEL wavelengths.

CSE is driven by the gradient of the bunch current and bunches with a steep rise of the electron density at the tail are preferable to drive the FEL. To create such bunches also having a reduced level of noise, we proposes the scheme that uses effects of noise reduction and controlled microbunching instability, and consists of a laser heater, a bunch compressor, and a shot noise suppression section. First, the bunch passes through the laser heater and bunch compressor. In our scheme the tail of the bunch is sensitive to the microbunching instability whereas the main core of the bunch is stable against the instability. This is realized by using a laser heater with a partial overlap between the electron and laser pulses such that the bunch tail remains unheated. Then, in the bunch compressor the bunch undergoes the longitudinal compression and the controlled microbunching of its tail occurs as well. We calculated the microbunching gain, $G(\lambda)$, with and without a laser heater and in the latter case $G(\lambda)$ attains its maximal value at a compressed wavelength of $2.7 \mu \mathrm{m}$. Therefore, if we modulate the bunch tail with a period of around $40 \mu \mathrm{m}$ (the compression factor is 16) by means of a photocathode laser (or laser modulator) and heat with the laser heater only the main core of the bunch leaving the tail unheated, then we can have the microbunched tail resulting in a steep variation of the electron density at the tail. There is enhancement of the microbunching in the vicinity of the resonant FEL wavelength so that the normal level of noise is preserved during magnetic compression. Therefore we use a shot noise suppression scheme downstream of the bunch compressor in order to reduce the level of shot noise. We found that in the compact noise suppression section of around $3 \mathrm{~m}$ the shot noise reduction by 2 orders of magnitude can be achieved for electron bunches produced by the SwissFEL injector. Thus, one can form quiet bunches capable of effective driving CSE in the FEL.

We have also analyzed a possible extension of the FEL based on CSE with a finite current gradient and reduced shot noise to the VUV region using the linear analytical theory developed in [18]. In fact, the FEL coherence depends on the ratio of the coherent and incoherent spontaneous emission, and if the latter is reduced, then dominance of CSE can be extended to shorter wavelengths. We estimated that VUV FELs based on self-amplified CSE would require the reduction of shot noise by 3 or 4 orders of magnitude in order to have radiation pulses with a welldetermined temporal structure. Simultaneously, the rise time of the current pulse should occur on a scale smaller than a few FEL resonant wavelengths. As the technological challenges on noise reduction are overcome, the proposed FEL may become an alternative to FELs using the regenerative amplifier concept or seeding schemes. Finally, CSE from quiet bunches is of interest not only for short wavelength FELs, but also infrared FELs may benefit from this approach. Moreover, an infrared FEL with moderately relativistic bunches can be a convenient test stand for studying properties of quiet bunches.

\section{ACKNOWLEDGMENTS}

The authors would like to acknowledge fruitful discussions with Dr. M. Dohlus, Dr. E. A. Schneidmiller, and Dr. S. Bettoni. We also express our gratitude to Professor A. Gover for explaining some elements of his theory. The authors are grateful to the referees for valuable comments and important suggestions. This publication was produced during V. A. Goryashko's tune at Uppsala University, thanks to the Swedish Institute for VISBY support.

\section{APPENDIX A: NUMERICAL IMPLEMENTATION}

To find a numerical solution to Eq. (11) let us introduce a uniform mesh over $\zeta$ with fixed step $\Delta \zeta$ such that the $k$ th node is located at $\zeta_{k}=k \Delta \zeta$. Then, we replace the integral from 0 to $\zeta$ by a sum of integrals over small intervals $\Delta \zeta$

$$
\int_{0}^{\zeta}(\ldots) \mathrm{d} \zeta^{\prime} \rightarrow \sum_{k=0}^{K} \int_{k \Delta \zeta}^{(k+1) \Delta \zeta}(\ldots) \mathrm{d} \zeta^{\prime},
$$

and expand $\tau_{q}\left(\zeta^{\prime}\right)$ into a Taylor series around $k \Delta \zeta$ up to the linear term

$$
\begin{aligned}
\tau_{q}\left(\zeta^{\prime}\right) & \approx \tau_{q}(k \Delta \zeta)+\left.\frac{d \tau q}{d \zeta^{\prime}}\right|_{\zeta^{\prime}=k \Delta \zeta}\left(\zeta^{\prime}-k \Delta \zeta\right) \\
& =\tau_{q}^{(k)}+2 \rho \mu_{q}^{(k)}\left(\zeta^{\prime}-k \Delta \zeta\right)
\end{aligned}
$$

where $\tau_{q}^{(k)}=\tau_{q}(k \Delta \zeta)$ and $\mu_{q}^{(k)}=\mu_{q}(k \Delta \zeta)$. Then, the field amplitude at the $K$ th node may be written as 


$$
\tilde{F}\left(\zeta_{K}, \tau\right)=\frac{1}{\bar{n}_{\|}} \sum_{k=0}^{K-1} \sum_{q=1}^{Q_{b}} \int_{k \Delta \zeta}^{(k+1) \Delta \zeta} \exp \left[-\frac{i\left(\tau+\zeta^{\prime}-K \Delta \zeta\right)}{2 \rho}\right] \times \chi_{q} \delta\left[\tau+\zeta^{\prime}-K \Delta \zeta-\tau_{q}^{(k)}-2 \rho \mu_{q}^{(k)}\left(\zeta^{\prime}-k \Delta \zeta\right)\right] d \zeta^{\prime} .
$$

Because of the Dirac Delta function, the integral over $\zeta^{\prime}$ is nonzero only if

$$
k \Delta \zeta \leq \zeta^{\prime}(\tau)<(k+1) \Delta \zeta
$$

where

$$
\zeta^{\prime}(\tau)=\frac{\tau-K \Delta \zeta+2 \rho \mu_{q}^{(k)} k \Delta \zeta-\tau_{q}^{(k)}}{2 \rho \mu_{q}^{(k)}-1}
$$

Note that $\zeta^{\prime}$ depends on time $\tau$, and we have to scan over $\tau$ to calculate the integral as a function of time. Hence, we introduce a mesh with respect to $\tau$ with step size $\Delta \tau$ and label nodes by subscript $j$ such that $\tau_{j}=j \Delta \tau$. When inequalities (A4) are fulfilled the field amplitude in position $\zeta_{k}$ at time $\tau_{j}$ reads

$$
\tilde{F}\left(\zeta_{K}, \tau_{j}\right)=\frac{1}{\bar{n}_{\|}} \sum_{k=0}^{K-1} \times \sum_{q=1}^{Q_{b}} \chi_{q} \exp \left[-\frac{i}{2 \rho} \frac{2 \rho \mu_{q}^{(k)}\left[\tau_{j}-(K-k) \Delta \zeta\right]-\tau_{q}^{(k)}}{2 \rho \mu_{q}^{(k)}-1}\right] .
$$

Note that $F\left(\zeta_{K+1}, \tau_{j}\right)$ and $F\left(\zeta_{K}, \tau_{j}\right)$ are related by

$$
\tilde{F}\left(\zeta_{K+1}, \tau_{j}\right)=\tilde{F}\left(\zeta_{K}, \tau_{j}-\Delta \zeta\right)+\frac{1}{\bar{n}_{\|}} \sum_{q=1}^{Q_{b}} \chi_{q} \exp \left[-\frac{i}{2 \rho} \frac{2 \rho \mu_{q}^{(K)} \tau_{j}-\tau_{q}^{(K)}}{2 \rho \mu_{q}^{(K)}-1}\right] .
$$

The last formula plays a critical role for fast numerical simulations without limitations on the computer memory since it allows one to keep in the computer memory only the $2 Q_{b}$ positions of electrons and the field generated at the previous step over $\zeta$.

The equations of motion are integrated using a combination of the predictor-corrector scheme and two-point AdamsBashforth-Moulton method [53]

$$
\begin{aligned}
\mu_{q}^{K+1 / 2} & =\mu_{q}^{K}-\Delta \zeta \operatorname{Re}\left\{\tilde{F}\left(\zeta_{K}, \tau_{q}^{K}\right) \mathrm{e}\left[\frac{i \tau_{q}^{K}}{2 \rho}\right]\left(1-\mathrm{e}^{i \zeta_{K} / \rho}\right)\right\}, \quad \tau_{q}^{K+1 / 2}=\tau_{q}^{K}+\Delta \zeta \rho \mu_{q}^{K}, \\
\mu_{q}^{K+1} & =\mu_{q}^{K}-\Delta \zeta \operatorname{Re}\left\{\exp \left[\frac{i \tau_{q}^{K+1 / 2}}{2 \rho}\right]\left(1-\mathrm{e}^{i \zeta_{K+1 / 2} / \rho}\right)\left[3 \tilde{F}\left(\zeta_{K}, \tau_{q}^{K+1 / 2}\right)-\tilde{F}\left(\zeta_{K-1}, \tau_{q}^{K+1 / 2}\right)\right]\right\}, \quad \tau_{q}^{K+1}=\tau_{q}^{K}+2 \Delta \zeta \rho \mu_{q}^{K+1 / 2}
\end{aligned}
$$

As we mentioned before, one needs to scan the condition (A4) over time to get the field amplitude as a function of time. Then, the question is what the time interval of scanning should be. Electrons enter the interaction region from $-T_{b}$ to 0 , and assuming a constant velocity one finds that the radiation field is nonzero at position $z$ if $t_{\text {pulse }}(z) \in$ $\left[z / c-T_{b}, z / \bar{v}_{\|}\right]$. In practice, this condition is exact since the tail of the bunch moves with constant average velocity $\bar{v}_{\|}$(the tail experiences no radiation field because of the slippage), but the head starts to radiate a field that propagates with the velocity of light. It is also clear that electron transit time $t_{q}$ is within the interval $t_{\text {pulse }}(z)$, and we need to calculate the field amplitude $F$ only for interval $t_{\text {pulse }}(z)$ at a given $z$. The corresponding interval for the dimensionless time is dictated by $\tau_{\min }=0$ and $\tau_{\max }=\zeta+\tau_{e}$. Thus, the developed technique has the advantage over finitedifference techniques $[11,54,55]$ in that the radiation field is calculated only over the time interval where it is nonzero, whereas the use of the finite-difference method for solving the excitation Eq. (11) would require one to calculate the field in all points of a mesh over interval $\tau \in$ $\left[0, \zeta_{\max }+\tau_{e}\right]$, thus increasing the calculation time and required computer memory. Moreover, if the full spacetime structure of the field is not needed, one can calculate the field only on a temporal mesh surrounding the bunch, thus decreasing the computation time even more.

\section{APPENDIX B: SHORT WAVELENGTH LIMIT OF CSE}

Let us estimate the bunch parameters needed for the operation of a FEL at short wavelengths (optical and shorter). A single pass FEL can be considered as a system, in which spontaneous radiation emitted by electron bunches in an undulator is subsequently amplified in a self-consisted way. Then, at the FEL startup one needs to achieve dominance of the coherent spontaneous emission over the incoherent spontaneous emission. The latter is 
dictated by the current shot noise, energy spread (it can also be categorized into the kinetic voltage noise and kinetic power noise as proposed by A. Gover [18]), and the "radiation noise," composed of quantum spontaneous emission and blackbody radiation at the FEL entrance [18]. Suppression of shot noise results in a reduction of the incoherent radiation by factor $g(S,\langle\Gamma\rangle)$ depending on the "radiation suppression parameter" $S$ and shot noise suppression parameter $\langle\Gamma\rangle)$; see details in [18]. Therefore, the total spontaneous radiated power at wave number $k$ may be estimated as

$$
P_{\text {spontaneous }}(k)=P_{\text {incoh }}(k) g(S,\langle\Gamma\rangle)+P_{\text {coh }}(k),
$$

where $P_{\text {incoh }}(k)=Q_{b} P_{\text {und }}(k)$ is the incoherent power corresponding to the normal level of shot noise and $P_{\text {coh }}(k)=$ $Q_{b}^{2}|F(k)|^{2} P_{\text {und }}(k)$ is the power of coherent emission. Here, $Q_{b}$ as before is the number of electrons in a bunch, $P_{\text {und }}(k)$ is the power of spontaneous radiation emitted by a single electron in the undulator, and $F(k)$ is the bunch form factor that at a given wavelength depends on the bunch length and the gradient of the bunch current. It attains its maximum value for bunches with a rectangular density distribution. Bunches with Gaussian or parabolic density distributions also result in CSE, but the radiation has noticeable levels only at long wavelengths and is normally vanishingly small beyond the infrared region [22].

In order to estimate requirements on the current gradient and noise reduction level, we consider the current pulse profile given by Eq. (15) and shown in Fig. 12. The form factor $F(k)$ is the Fourier transform of the current profile, and the absolute value of $F(k)$ squared reads

$$
|F(k)|^{2}=\left|F_{\text {rec }}(k)\right|^{2}\left|F_{\text {fron }}(k)\right|^{2},
$$

where

$$
\left|F_{\text {rec }}(k)\right|^{2}=\left(\frac{\sin \left[k L_{b} / 2\right]}{k L_{b} / 2}\right)^{2}
$$

is the form factor of the rectangular pulse and

$$
\left|F_{\text {fron }}(k)\right|^{2}=\left(\frac{\pi^{2} \cos [k \Delta \lambda / 2]}{\pi^{2}-k^{2} \Delta \lambda^{2}}\right)^{2}
$$

takes into account the finite rise time. Typically $k L_{b} \gg 1$, then the condition of dominance of the coherent emission over an incoherent one reads

$$
\frac{Q_{b}\left|F_{\text {fron }}(k)\right|^{2}}{\left(k L_{b}\right)^{2}} \gg g[S,\langle\Gamma\rangle] .
$$

The radiation suppression parameter $S$ is determined by the electron bunch collective dynamics in the transport line and is a complicated function of bunch parameters [18], but for an order of estimate we may use $S \sim \lambda_{u} /\left(\lambda_{p} \rho\right)$. Recall that $\lambda_{u}$ and $\lambda_{p}$ are the undulator and plasma wavelengths, respectively, and $\rho$ is the FEL parameter. As the wavelength decreases, $F(k)$ also decreases but the condition
(B5) can remain fulfilled on the expense of the shot noise reduction. Thus, the principal question is to what frequencies the noise suppression scheme can be exploited. Papers $[18,21,56]$ suggest that noise can be suppressed up to the VUV spectral range. For the noise suppression scheme proposed by A. Gover, we made estimates for a wavelength of $50 \mathrm{~nm}$ and found that noise reduction is $10^{-4}$ and the radiation suppression parameter is $10^{-5}$; therefore the incoherent power is reduced by $g=(\langle\Gamma\rangle / S)^{2} \sim 10^{-2}$. At the same time, $\left|F_{\text {rec }}(k)\right|^{2}$ is also decreased by 2 orders of magnitude as compared to the case considered in our paper. If the decrease of the left-hand side of Eq. (B5) caused by $\left|F_{\text {front }}(k)\right|^{2}$ is not essential, then we may expect the dominance of the coherent emission over the incoherent one for VUV FEL to be of the same order of magnitude as considered in our paper for the case $\langle\Gamma\rangle \sim 1$. The factor $\left|F_{\text {front }}(k)\right|^{2}$ is proportional to $1 /(k \Delta \lambda)^{4}$ for $k \Delta \lambda \gg 1$ and would eliminate CSE if the gradient is small such that we need to require $k \Delta \lambda \sim 1$ in order to preserve the dominance of the coherent emission over the incoherent one. This means that the rise time of the current pulse should occur on a scale of several FEL resonant wavelengths. As proposed in this paper, sharp density modulations of the bunch tail can be obtained by means of the controlled microbunching instability in a way similar to that employed in LSCA. Formation of such modulations in the VUV region by means of the controlled microbunching instability does not require strong wavelength compression such that tolerances for the chirp stability are relaxed and we expect no fundamental problems with the bunch formation.

[1] B. W. J. McNeil and N. R. Thompson, Nat. Photonics 4, 814 (2010).

[2] E. L. Saldin, E. A. Schneidmiller, and M. V. Yurkov, New J. Phys. 12, 035010 (2010).

[3] N. R. Thompson, D. J. Dunning, B. W. J. McNeil, and R. Bartolini, Proceedings of the 31st International Free Electron Laser Conference (FEL 09), Liverpool, UK (STFC Daresbury Laboratory, Warrington, 2009).

[4] L. Yu, Phys. Rev. A 44, 5178 (1991).

[5] G. Stupakov, Phys. Rev. Lett. 102, 074801 (2009).

[6] J. Amann et al., Nat. Photonics 6, 693 (2012).

[7] L. Giannessi et al., Phys. Rev. Lett. 106, 144801 (2011).

[8] A. Doria, R. Bartolini, J. Feinstein, G. P. Gallerano, and R. H. Pantell, IEEE J. Quantum Electron. 29, 1428 (1993).

[9] D. A. Jaroszynski, R. J. Bakker, A. F. G. V. D. Meer, D. Oepts, and P.W. V. Amersfoort, Phys. Rev. Lett. 71, 3798 (1993).

[10] S. Krinsky, Phys. Rev. E 59, 1171 (1999).

[11] B. W. J. McNeil, G. R. M. Robb, and D. A. Jaroszynski, Opt. Commun. 165, 65 (1999).

[12] A. Gover and E. Dyunin, Phys. Rev. Lett. 102, 154801 (2009). 
[13] V. N. Litvinenko, Proceedings of the 31st International Free Electron Laser Conference (FEL 09), Liverpool, UK (Ref. [3]).

[14] D. Ratner, Z. Huang, and G. Stupakov, Phys. Rev. ST Accel. Beams 14, 060710 (2011).

[15] P. Musumeci, R. K. Li, and A. Marinelli, Phys. Rev. Lett. 106, 184801 (2011).

[16] D. Ratner and G. Stupakov, Phys. Rev. Lett. 109, 034801 (2012).

[17] A. Gover, A. Nause, E. Dyunin, and M. Fedurin, Nat. Phys., 8, 877 (2012).

[18] A. Gover and E. Dyunin, IEEE J. Quantum Electron. 46, 1511 (2010).

[19] A. G. E. Dyunin and A. Nause, IEEE Trans. Plasma Sci. 39, 2033 (2011).

[20] K.-J. Kim and R. Lindberg, Proceedings of the 33rd International Free Electron Laser Conference (FEL 2011), Shanghai, China (Shanghai Institute of Applied Physics, Shanghai, Chine, 2011), p. TUOA2

[21] K.-J. Kim, "Irreducible Quantum and Classical Noise in High-Gain FEL Amplifier," 4th Microbunching Workshop, College Park, MD, USA, 2012, www.umer.umd.edu/ events_folder/uBi12/ubi12- Talks/ubi12-Session2.

[22] B. W. J. McNeil and G. R. M. Robb, Phys. Rev. E 65, 046503 (2002).

[23] D. Bocek, P. Kung, H.C. Lihn, C. Settakorn, and H. Wiedemann, Nucl. Instrum. Methods Phys. Res., Sect. A 375, 13 (1996).

[24] Lawrence Berkeley National Laboratory, White Paper, Report No. SLAC-R- 910 and SLAC National Accelerator Laboratory, White Paper, Report No. LBNL1090E, edited by A. L. Robinson and B. Plummer.

[25] G. Cerullo and S. D. Silvestri, Rev. Sci. Instrum. 74, 1 (2003).

[26] T. Zhang, Z. Zhao, and X. Yang, Proceedings of the 3rd International Particle Accelerator Conference, New Orleans, Louisiana, USA, 2012 (IEEE, Piscataway, NJ, 2012), TUPPP057.

[27] "Realizing the Potential of Seeded FELs in the Soft X-Ray Regime," Final Report, SLAC and LBNL Workshop, 2011.

[28] Workshop on the Science and Technology of 4th Generation Light Sources Based on Superconducting Technology (Stockholm University, Sweden, 2012).

[29] S. G. Biedron, S. V. Milton, and H.P. Freund, Nucl. Instrum. Methods Phys. Res., Sect. A 475, 401 (2001).

[30] T. Shintake, Proceedings of the 29th International Free Electron Laser Conference (FEL 2007), Novosibirsk, Russia (Budker Institute of Nuclear Physics, Novosibirsk, Russia, 2007), WEPPH021. Earlier internal report cited here: T. Shintake, KEK Internal Report, AccelLab-99-1 (2007).

[31] T. Shaftan and L.H. Yu, Phys. Rev. E 71, 046501 (2005).

[32] D. Ratner, A. Chao, and Z. Huang, Phys. Rev. ST Accel. Beams 14, 020701 (2011).

[33] G. Geloni, V. Kocharyan, and E. Saldin, arXiv:1111.1615v1.
[34] E. L. Saldin, E. A. Schneidmiller, and M. V. Yurkov, Nucl. Instrum. Methods Phys. Res., Sect. A 490, 1 (2002).

[35] S. Heifets, G. Stupakov, and S. Krinsky, Phys. Rev. ST Accel. Beams 5, 064401 (2002).

[36] E. A. Schneidmiller and M. V. Yurkov, Phys. Rev. ST Accel. Beams 13, 110701 (2010).

[37] E. L. Saldin, E. A. Schneidmiller, and M. V. Yurkov, Nucl. Instrum. Methods Phys. Res., Sect. A 528, 355 (2004).

[38] Z. Huang, M. Borland, P. Emma, J. Wu, C. Limborga, G. Stupakov, and J. Welch, Phys. Rev. ST Accel. Beams 7, 074401 (2004).

[39] A. D. Polyanin, Handbook of Linear Partial Differential Equations for Engineers and Scientists (Chapman and Hall, London, UK, 2001).

[40] A. B. McNeil, A. G. Robb, A. D. Dunning, and A. N. Thompson, Proceedings of the 28th Free Electron Laser Conference (FEL 06), Berlin, Germany, 2006 (BESSY, Berlin, Germany, 2006), MOPPH011.

[41] R. Bonifacio, B. W. J. McNeil, and P. Pierini, Phys. Rev. A 40, 4467 (1989).

[42] M. Xie, Proceedings of the Particle Accelerator Conference, Dallas, TX, 1995 (IEEE, New York, 1995), Vol. 2, p. 183.

[43] Z. Huang and K. Kim, Phys. Rev. ST Accel. Beams 10, 034801 (2007).

[44] E. L. Saldin, E. A. Schneidmiller, and M. V. Yurkov, The Physics of Free Electron Lasers (Springer, Berlin, Germany, 2000).

[45] L. Giannessi, Phys. Rev. ST Accel. Beams 6, 114802 (2003).

[46] B. W. J. McNeil, M. W. Poole, and G. R. M. Robb, Phys. Rev. ST Accel. Beams 6, 070701 (2003).

[47] S. I. C. D. Report, www.psi.ch/swissfel.

[48] We are grateful to one of the referees for pointing this out.

[49] One of the referees pointed out that a shot noise suppression section has to be downstream a bunch compressor but not upstream because the bunch compressor washes out a reduced level of shot noise.

[50] S. Seletskiy, Y. Hidaka, J. B. Murphy, B. Podobedov, H. Qian, Y. Shen, X. J. Wang, and X. Yang, Phys. Rev. ST Accel. Beams 14, 110701 (2011).

[51] S. Bettoni, B. Beutner, and V. A. Goryashko, Proceedings of the 3rd International Particle Accelerator Conference, New Orleans, Louisiana, USA, 2012 (IEEE, Piscataway, $\mathrm{NJ}, 2012$ ).

[52] K. K.Z. Huang, Phys. Rev. ST Accel. Beams 5, 074401 (2002).

[53] D. Potter, Computational Physics (John Wiley \& Sons Ltd., New York, USA, 1973).

[54] C. Maroli, V. Petrillo, and M. Ferrario, Phys. Rev. ST Accel. Beams 14, 070703 (2011).

[55] S. I. Bajlekov, W. M. Fawley, C. B. Schroeder, R. Bartolini, and S. M. Hooker, Phys. Rev. ST Accel. Beams 14, 060711 (2011).

[56] E. Dyunin, A. Nause, and A. Gover, IEEE Trans. Plasma Sci. 39, 2033 (2011). 